\title{
Clinical Analysis of Neonatal Food Protein Induced Enterocolitis Syndrome in 137 Newborn Infants

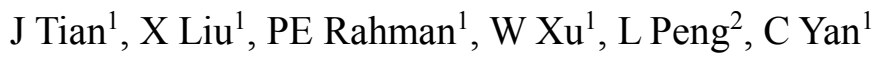

\begin{abstract}
Objective: To explore the clinical characters, diagnosis, therapy and prognosis of neonatal food protein induced enterocolitis syndrome (NFPIES) to provide a reference for diagnosis and treatment.
\end{abstract}

Methods: 137 newborn infants admitted to the neonatology department of our hospital between 2007 and 2012 were included in the study and an analysis of the gender, season of admission, delivery history, clinical manifestation, stool test, $\mathrm{C}$ Reactive Protein and application of antibiotics was performed.

Results: There was a gradual increase in morbidity, year over year, in NFPIES infants admitted to the neonatology department between 2007 and 2012. Fetal factors: Birth weight between 2.5 and $4 \mathrm{kgs}$ played a significant role. NFPIES occurs between days 1 to 28 birth with a median of 16 days. The number of infants that developed NFPIES within 14 days of birth was 63 (46\%). The variation of $\mathrm{C}$ reactive protein was between $0.15 \mathrm{mg} / \mathrm{l}$ and $164 \mathrm{mg} / \mathrm{l}$ (with an average of $18.82 \mathrm{mg} / \mathrm{l}$ ) in the 137 newborn infants in the study. 36 infants recovered without the application of antibiotics and were termed as Group A and the other 101 cases were called Group B. The duration of recovery for group A was shorter than that for Group B $(p<0.05)$.

Conclusion: In the past 6 years, the number of NFPIES cases admitted to the neonatology department of our hospital presents a rising trend. NFPIES occurred mainly among term infants, those with mixed feeding, caesarean section and in infants with normal birth weight. The clinical manifestation of NFPIES was not typical, so the diagnosis and differential diagnosis were difficult.

Keywords: Allergy, enterocolitis syndrome, neonatal food protein induction, newborn infants From: ${ }^{1}$ Department of Neonatology and ${ }^{2}$ Department of Respiration, The First Bethune Hospital of Jilin University, Changchun, Jilin 130021, China.

Correspondence: Dr C Yan, Department of Neonatology and Dr L Peng, Department of Respiration, The First Bethune Hospital of Jilin University, No. 71, Xinmin Street, Changchun, Jilin 130021, China. Fax: +86-0431-88782222, email:chaoyingyan9988@sohu.com, lipingpeng0116@sina.com 


\section{INTRODUCTION}

Neonatal Food Protein Induced enterocolitis syndrome (NFPIES) is a type of gastrointestinal disease in newborn infants, especially in term infants with normal birth weight. It is a non- $\operatorname{IgE}$ mediated food allergy syndrome that is rare and its symptoms are confined to the gastrointestinal system (1). The major clinical syndromes include vomiting, abdominal distension, diarrhea, blood in stool, crying after being fed, which would result in the occurrence of malnutrition, stenosis of bowel and necrotizing enterocolitis (NEC) if not diagnosed or treated in a timely fashion. The pathophysiological mechanisms are not yet fully understood and its incidence is difficult to ascertain because of frequent misdiagnosis. The common cause of NFPIES is cow's milk (2). Cases have been reported as early as 1940 with intestinal bleeding in newborns due to cow's milk allergy (3). Gryboski (4) described infants presenting in the first 6 weeks of life with recurrent vomiting, bloody diarrhea, and abdominal distension while being fed with cow's milk-based formula $(4,5)$.

In the most severe cases, symptoms start within first days of life and abdominal radiographs show intramural gas often leading to a diagnosis of NEC followed by antibiotic administration (6). Current therapy is based on intravenous fluid replacement and steroid administration, while oral rehydration may be sufficient in mild cases. Although the differential diagnosis of NFPIES has been considered an important issue for many years misdiagnosis may delay the identification of NFPIES for months, exposing the children to the repetition of acute episodes (7-8). Therefore, the application of antibiotics prolonged the course of disease, or the application of relative derived antibiotics resulted in complications. 
Identification of the risk factors of NFPIES during the neonatal period is of clinical significance in seeking strategies for early diagnosis and treatment.

\section{MATERIALS AND METHODS}

137 newborn infants with NFPIES admitted to neonatology department of our hospital from 2007 to 2012 and conforming to the inclusion criteria, and making analysis of these cases.

\section{Inclusion criteria}

1) As for NFPIES, it is according to the clinical diagnostic criteria recommended by World Allergy Organization (WAO) Diagnosis and Rationale for Action against Cow's Milk Allergy (DRACMA) Guidelines; as for the complications, it is diagnosed and treated according to Practical Neonatology (the $4^{\text {th }}$ edition) related diagnostic criteria.

2) The sick newborn infants that were considered as other infectious diseases when admitted to hospital, but the efficacy was poor or the disease was aggravated after taking antibiotics, and the syndrome was improved after stopping antibiotics, fasting, food avoiding, taking depth hydrolyzed formula milk powder.

3) Being attacked within 28 days after birth.

\section{RESULTS}

\section{Clinical characteristics}

From Jan. 2007 to Dec. 2012, 137 newborn infants with NFPIES, admitted to neonatology 
department of The First Bethune Hospital of Jilin University that conformed to the inclusion criteria were chosen for this study. During the 6 years, the morbidity of NFPIES demonstrated a trend of increase year over year, as shown in Table 1. The onset of NFPIES ranged between 1 and 28 days after birth and the median was 16 days. Of the 132 subjects, $105(82 \%)$ were infected within 14 days after birth. There were 112 term infants (82\%), 23 premature infants (16.7\%) and 2 post-term infants $(1.3 \%)$, as shown in Table 2 . Of the 137 infants, 84 were males s (61\%) and 53 were females (39\%).

\section{NFPIES related factors}

Gender, weight and mode of delivery

There were 103 cases (75\%) with birth weight between 2.5 and $4 \mathrm{kgs}, 28$ cases (20.8\%) with birth weight between 1 and $2.5 \mathrm{kgs}$ and 6 cases (4.2\%) with birth weight over $4 \mathrm{kgs}$, as shown in Table 3. There were 96 cases (70.1\%) delivered by caesarean section and 41 cases $(29.9 \%)$ were by normal vaginal delivery.

\section{Morbidity by age in days, feeding patterns, seasonal factors and clinical manifestation}

The morbidity was determined between 1 to 28 days after birth (with a median of 16 days) and the results showed that 63 cases (46\%) developed NFPIES within 14 days after birth. Among the 137 cases in the study, $73(52.8 \%)$ were infants receiving mixed feeding, 36 (26.4\%) received artificial feeding and $28(20.8 \%)$ were breast fed infants. Examination of seasonal factors demonstrated that $27(19.7 \%)$ were born in spring, $46(33.5 \%)$ in summer, 38 $(27.7 \%)$ in autumn and $26(19.1 \%)$ were born in winter (Table 4). As to the clinical manifestation, there were 74 cases $(54 \%)$ of diarrhea and abdominal distention, which 
comprised the largest group, while the number of cases presenting with vomiting, milk rejecting and blood in stool decreased progressively.

\section{Family history}

There were 30 infants $(22 \%)$ with one parent having allergic rhinitis, asthma, contact dermatitis or urticaria and drug or food allergies. The distribution of which was 19 cases of allergic rhinitis, 7 cases of asthma, 3 cases of contact dermatitis and 1 case of penicillin anaphylaxis.

\section{History of antibiotic therapy}

Of the 137 infants chosen in this study, 36 that did not receive antibiotics were regarded as Group A and the other 101 cases were regarded as Group B. Observation of the two groups showed a similar state of illness but different concomitant diseases and the results are as shown in Table 5 and Table 6.

Group B was divided into two sub-groups according to the duration of antibiotic administration. 7 days was chosen as the dividing criteria, with less than 7 days administration as one sub-group and greater than 7 days as the other sub-group, so as to observe the distribution of neonatal infection in the two groups. The results are as shown in Table 7.

\section{Laboratory examination}

Variations in the level of C-reactive protein were between $0.15 \mathrm{mg} / 1-164 \mathrm{mg} / 1$ (with an average of $18.82 \mathrm{mg} / \mathrm{l})$. Stool test showed that 35 cases $(26 \%)$ were positive for fat droplet and 23 cases $(16.8 \%)$ were positive for occult blood. 


\section{Concomitant diseases}

We examined the occurrence of concomitant diseases among the 137 infants and found that $79 \%(108)$ of the cases presented with comorbidities. We found that anemia $(50.4 \%)$ and infection $(24.8 \%)$ were the two most common concomitant conditions in the infants suffering from NFPIES, as shown in Table 8. Hyponatremia, NEC and hypoglycemia were the other comorbidities found but were restricted to a few cases.

\section{DISCUSSION}

Food protein induced gastroenteropathy is a type of IgE-mediated allergic disease, which is also called food allergic gastroenteropathy. Non-IgE-mediated food allergic gastroenteropathy includes food protein induced enterocolitis syndrome, and the allergic symptom is limited to the course of disease as sub-acute or chronic gastroenteropathy (9). Enterocolitis syndrome mainly appears in the neonatal period and in early infancy (10). NFPIES refers to a common abdominal immune reaction in newborn infants.

In this study, the morbidity of NFPIES among the infants admitted to the neonatology department in our hospital, showed a trend of gradual increase year over year for the past 6 years. Some of the contributing factors could be urbanization, wide spread immunization, over use of antibiotics and air pollution. In addition, environmental pollution may result in defects in the development of the immune system leading to immune disorders and also affect normal immune response. NFPIES can occur at any time during the neonatal period and it commonly occurs 1 week after birth. The 7-14 day period following birth is the 
time for in vivo steady state recovery. With a gradual increase in enteral feeding volume, the intestinal tract begins to receive larger heterologous proteins and this could lead to feeding intolerance and allergies.

The relationship between morbidity of allergic diseases and birth season has been perceived. Kuzume et al.indicated that there is a certain relationship between morbidity of allergic diseases and climatic conditions at the time of birth, according to a study referring to birth season, morbidity of allergic diseases and allergy-related hematological examination (11). The research results of the morbidity of atopic dermatitis in infants indicated that the morbidity was low in infants born during the spring season and high in infants born in autumn. They considered that the variation was related to parental gene expression influenced by ultraviolet irradiation. The result of this study was not in complete consensus with that of Kazuyo's report which involved 46 infants $(33.5 \%)$ born in summer, and then $38(27.7 \%)$ in sutumn. Though the difference may have resulted from the small sample size of the study, the overall results indicated that the morbidity of NFPIES was high in infants born during the warmer months.

Compared with natural delivery, infants delivered by cesarean did not come in contact with normal parental flora of the birth canal. Administration of antibiotics and delayed breast feeding in infants delivered by cesarean section, resulted in them facing more allergic risks compared with those born naturally. In addition, the proportion of bacteria in the stool samples of infants, delivered by cesarean, also proved to be different. It was considered that Th1 type of immune response was activated when infants came in contact with parent vaginal 
flora during birth, which could promote a relative steady state between Th1 and Th2 immune responses.

There has been a previous study on newborn infants with high allergic risks during delivery, where three groups of infants were followed up. The results demonstrated that ordinary cow milk group developed more allergic diseases. The specific functions of human milk in newborn infants had been confirmed, and human milk contains substances that are absent in other animals, such as long chain polyunsaturated fatty acid, digestible protein and immune ingredients, which play a role in the anti-inflammatory and immune regulation function to protect gastrointestinal mucous membrane and thus promoting maturation of gastrointestinal tract. Interestingly, the osmotic pressure of breast milk is lower than that of formula milk, which can reduce the risk of NFPIES.

It was reported that the risk of onset of NFPIES, in newborn infants, with both parents having history of allergy, was $62 \%$ and for those with one parent having history of allergy was $37 \%$. Therefore, family history of allergy could be indicative of the onset of allergic diseases in newborn infants. Some scholars had also found that in drug allergy studies, genes such as HLA-B5701 presented certain relations between drug and allergy (12).

More than $70 \%$ immune cells in the human body exist in the intestinal mucosa and have a certain relation with intestinal flora (13-14). The number of intestinal bacteria is more than the total number of bacteria in other parts of the body. The function of bacterial colony formation in the intestinal tract in immune development has been confirmed using animal models (15). However, mice living in sterile environment after birth cannot induce food 
tolerance. Antibiotic application without any indication of infection would induce maladjustment of intestinal flora and further aggravate the occurrence of feeding intolerance and diarrhea and could result in fungal infection in severe cases. However, combined infection could not be excluded in the cases. Therefore, correct diagnosis and reasonable administration of antibiotics seems to be especially important.

CRP is a non-specific inflammatory reactant of tissue injury. It has been found in previous studies that factors such as the sedimentation of virus, bacteria, obstruction and immune complex could result in tissue injury (16). As CRP is transmitted, through the placenta, from mother to fetus during birth, examination of CRP 3 days after birth would be of clinical significance. As for newborn infants, both sensitivity and specificity of CRP were very poor. In this study, the results showed that the range of CRP was wide $(0.15 \mathrm{mg} / 1-164 \mathrm{mg} / \mathrm{l})$. As the NFPIES is an immuno-reactive disease, the degree of elevation of CRP level was related to the immune state of infant. Therefore, there were certain limitations to considering CRP level as the indicator of bacterial infection.

In stool test of 137 cases, there were 35 cases $(26 \%)$ that were positive for fat droplet and 23 cases $(16.8 \%)$ positive for occult blood. Bloody stool was considered as a specific symptom for neonatal enteritis anaphylactica, which was diagnosed in the clinic but not consistent with laboratory examination. The manifestation was significant upon observation under endoscope. Allergy is usually caused by contact between gastrointestinal mucosa and protein, which can be seen as slight errhysis on the surface under endoscopy, with scarlatina hemorrhagic spot, fragile mucous membrane that is easy to bleed on touched. Several severe 
cases presented with hematocele under gastrointestinal mucosa with local apophysis to form kermesinus hematoma. X-ray of the abdomen demonstrated unevenness of pneumatosis in the intestinal canal, with dilatation of parts of the intestine but with no particular specificity.

Of the 137 NFPIES cases in this study, 108 infants (78.8\%) presented with related concomitant diseases, which were neonatal anemia, infection, hyponatremia, NEC and hypoglycemia, according to the morbidity from high to low, respectively. Under strong anaphylactic reaction, the levels of pro-inflammatory factors such as IL-1, 6, 8, 10 and tumor necrosis factor (TNF)- $\alpha$ were enhanced, while the functions of anti-inflammatory factors such as IL-4 and its receptors were weakened, which caused cascade amplification inflammatory reactions that resulted in ischemia and anoxia of intestinal wall and even perforation in severe cases and finally induced the onset of NEC. With more studies being done on NFPIES, it is being regarded as one of the reasons for the onset of NEC.

\section{Therapy}

Mild to moderate cases of NFPIES with breast feeding could continue this mode of feeding, but the mother should avoid taking eggs and milk for 2-4 weeks and supplement calcium, simultaneously. The observation should be continued, and if symptoms improved, the mother could take CMP. If symptoms re-appeared, the infants should be fed with depth hydrolyzed formula milk powder for 2-4 weeks after ablactation. Infants in whom the symptoms improved could be fed formula milk powder and gradually introduced to CMP and followed up. If symptoms re-appeared, depth hydrolyzed formula milk powder or special amino acid milk powder could be fed for 9-12 months. If the symptoms did not improve after the infants 
were fed with breast milk or formula milk powder, the mother could return to normal diet and other diagnosis should be considered.

As to infants suspected of severe NFPIES, if they presented with diarrhea or regurgitation, vomit, malnutrition, milk rejection or moderate-to-severe hematochezia induced hemoglobin reduction, they should be diagnosed and treated by clinicians in neonatology department and the mother should avoid allergenic diet. There had been many studies confirming that application of probiotics or prebiotics could complement normal flora and produce endogenous antibodies to compete for adhesion sites with pathogenic bacteria (17-19). Interestingly, inflammatory factors and media could also be used to modulate the immune system to reduce translocation of intestinal bacteria, which could effectively decrease the morbidity of NAE.

\section{CONCLUSION}

In the clinical study of pro-biotic application, there were no adverse effects or pro-biotic induced infections reported (20). Therefore, pro-biotic application should be considered as conventional therapy for premature infants.

\section{ACKNOWLEDGMENTS}

This study doesn't have any grant supported. 


\section{AUTHOR CONTRIBUTIONS}

Paraty Erkin Rahman participated in its design and wrote this paper, Xuehua Liu performed the statistical analysis and conceived of the study, Wei Xu read and approved this study.The authors declare that they have no conflicts of interest. 


\section{REFERENCES}

1. Katz Y, Goldberg MR, Rajuan N, Cohen A, Leshno M. The prevalence and natural course of food protein-induced enterocolitis syndrome to cow's milk: a large-scale, prospective population-based study. J Allergy Clin Immunol 2011; 127: 647-53.

2. Caubet JC, Ford LS, Sickles L, Järvinen KM, Sicherer SH, Sampson HA, et al. Clinical features and resolution of food protein-induced enterocolitis syndrome: 10-year experience. J Allergy Clin Immunol 2014; 134:382-9.

3. Rubin MI. Allergic intestinal bleeding in the newborn; a clinical syndrome. Am J Med Sci 1940; 200:385-90.

4. Gryboski JD. Gastrointestinal milk allergy in infants. Pediatrics 1967; 40:354-62.

5. Powell GK. Milk- and soy-induced enterocolitis of infancy. Clinical features and standardization of challenge. J Pediatr 1978; 93:553-60.

6. Nowak-Węgrzyn A, Sampson HA, Wood RA, Sicherer SH. Food protein-induced enterocolitis syndrome caused by solid food proteins. Pediatrics 2003; 111:829-35.

7. Powell GK. Food protein-induced enterocolitis of infancy: differential diagnosis and management. Compr Ther 1986; 12:28-37.

8. Leonard SA. Food protein-induced enterocolitis syndrome: an update on natural history and review of management. Ann Allergy Asthma Immunol 2011; 107:95-101

9. Maloney J, Nowak-Wegrzyn AE. Educational clinical case series for pediatric allergy and immunology: allergic proctocolitis, food protein-induced enterocolitis syndrome and allergic eosinophilic gastroenteritis with protein-losing gastroenteropathy as 
manifestations of non-IgE-mediated cow's milk allergy.Pediatr Allergy Immunol 2007; 18: 360-7.

10. Sicherer SH, Eigenmann PA, Sampson HA. Clinical features of food protein-induced enterocolitis syndrome.1998; 133: 214-9.

11. Kuzume K, Kusu M. Before-birth climatologic data may play a role in the development of allergies in infants. Pediatr Allergy Immunol. 2007; 18: 281-7.

12. Stekler J, Maenza J, Stevens C, Holte S, Malhotra U, McElrath MJ, et al. Abacavir hypersensitivity reaction in primary HIV infection. AIDS. 2006; 20: 1269-74.

13. Waters LJ, Mandalia S, Gazzard B, Nelson M. Prospective HLA-B*5701 screening and abacavir hypersensitivity: a single centre experience. AIDS. 2007; 21: 2533-4.

14. Vighi G, Marcucci F, Sensi L, Di Cara G, Frati F. Allergy and the gastrointestinal system. Clin Exp Immunol. 2008; 153: 3-6.

15. Tlaskalová-Hogenová H, Stěpánková R, Kozáková H, Hudcovic T, Vannucci L, Tučková L et al. The role of gut microbiota (commensal bacteria) and the mucosal barrier in the pathogenesis of inflammatory and autoimmune diseases and cancer: contribution of germ-free and gnotobiotic animal models of human diseases. Cell Mol Immunol. 2011; 8: 110-20.

16. Castro C, Gourley M. Diagnostic Testing and Interpretation of Tests for Autoimmunity. J Allergy Clin Immunol 2010; 125: S238-47.

17. Limdi JK, O’Neill C, McLaughlin J. Do probiotics have a therapeutic role in gastroenterology? World J Gastroenterol.2006; 12: 5447-57. 
18. Fiocchi A, Burks W, Bahna SL, Bielory L, Boyle RJ, Cocco R et al. Clinical Use of Probiotics in Pediatric Allergy (cuppa): A World Allergy Organization Position Paper. World Allergy Organ J.2012; 5: 148-67.

19. Sheridan PO, Bindels LB, Saulnier DM, Reid G, Nova E, Holmgren K et al. Can prebiotics and probiotics improve therapeutic outcomes for undernourished individuals? Gut Microbes.2014; 5: 74-82.

20. Sanders ME, Akkermans LMA, Haller D, Hammerman C, Heimbach J, Hörmannsperger $G$ et al. Safety assessment of probiotics for human use. Gut Microbes.2010; 1: 164-85. 
Table 1: Distribution of morbidity in infants with NFPIES

\begin{tabular}{lcccc}
\hline Year & $\begin{array}{l}\text { NFPIES } \\
\text { (case) }\end{array}$ & case & $\begin{array}{r}\text { number } \\
\text { Total case number admitted }\end{array}$ & $\begin{array}{r}\text { Constitue } \\
\text { int ratio (\%o) }\end{array}$ \\
\hline 2007 & 11 & 2356 & 4.67 \\
2008 & 17 & 3215 & 5.29 \\
2009 & 22 & 3538 & 6.21 \\
2010 & 23 & 3660 & 6.28 \\
2011 & 28 & 3984 & 7.01 \\
2012 & 36 & 4362 & 8.25 \\
Total & 137 & & 6.49 \\
\hline
\end{tabular}

Table 2: Constituent ratios of gestational age of NFPIES cases

\begin{tabular}{lcc}
\hline Gestational age & Cases & Percentage \\
\hline Term infants & 112 & $82 \%$ \\
Premature infants & 23 & $16.7 \%$ \\
Post-term infants & 2 & $1.3 \%$ \\
\hline
\end{tabular}


Table 3: Distribution of birth weight of NFPIES cases

\begin{tabular}{lcc}
\hline Weight $(\mathbf{k g})$ & Cases & Percentage \\
\hline $2.5-4.0$ & 103 & $75 \%$ \\
$1.0-2.49$ & 28 & $20.8 \%$ \\
$>4.0$ & 6 & $4.2 \%$ \\
\hline
\end{tabular}

Table 4: Clinical manifestations

\begin{tabular}{lcc}
\hline Clinical Manifestation & Cases & Percentage \\
\hline Diarrhea and abdominal distention 74 & $54 \%$ \\
Vomiting & 27 & $19.7 \%$ \\
Milk rejecting & 19 & $13.9 \%$ \\
Blood in stool & 17 & $12.4 \%$ \\
\hline
\end{tabular}

Table 5: Differences in concomitant diseases among the two groups [ $\mathrm{n} \%]$

\begin{tabular}{lcc}
\hline Group & Group A & Group B \\
\hline Neonatal anemia & $18(50 \%)$ & $51(50.1 \%)$ \\
Neonatal infection & $9(25 \%)$ & $25(24.8 \%)$ \\
$\chi^{2}$ & & 0.00 \\
$P$ & & $>0.05$ \\
\hline
\end{tabular}

Note: $p>0.05$, there was no difference between the two groups as to neonatal anemia and neonatal infection. 
Table 6: Number of cases and remission time in different groups $(\bar{x} \pm \mathrm{s})$

\begin{tabular}{cc}
\hline Group & Average remission days \\
\hline $\mathrm{A}(\mathrm{n}=36)$ & $5.57 \pm 2.41$ \\
$\mathrm{~B}(\mathrm{n}=101)$ & $9.13 \pm 4.31$ \\
$t$ & -3.46 \\
$P$ & $<0.05$
\end{tabular}

Note: The average remission days was calculated using t-test of two independent samples from Group A and B and the $\mathrm{t}$ value was $-3.463(p<0.05)$ which was a statistically significant difference.

Table 7: The effect of antibiotic application duration in the distribution of diseases [n (\%)]

\begin{tabular}{ccc} 
Group & Neonatal infection & Non-neonatal infection \\
\hline$\leq 7$ days & $6(5.9 \%)$ & $39(38.6 \%)$ \\
$>7$ days & $28(27.7 \%)$ & $28(27.7 \%)$ \\
$\chi^{2}$ & & 15.02 \\
$P$ & & $<0.01$
\end{tabular}

Note: chi-square value was 15.02 and $p<0.01$. The distribution of antibiotic application duration $>7$ days and $<7$ days were of statistical significance in neonatal infection.

Table 8: Concomitant diseases of NFPIES

\begin{tabular}{lcc}
\hline Concomitant diseases & Cases (n) & Percentage (\%) \\
\hline Neonatal anemia & 69 & $50.4 \%$ \\
Neonatal infection & 34 & $24.8 \%$ \\
Neonatal hyponatremia & 3 & $2.2 \%$ \\
NEC & $\mathbf{1}$ & $\mathbf{0 . 7 \%}$ \\
Hypoglycemia & $\mathbf{1}$ & $\mathbf{0 . 7 \%}$ \\
\hline
\end{tabular}

